\title{
Various complexity measures in confined hydrogen atom
}

\author{
Sangita Majumdar, Neetik Mukherjee, and Amlan K. Roy* \\ Department of Chemical Sciences \\ Indian Institute of Science Education and Research (IISER) Kolkata, \\ Mohanpur-741246, Nadia, WB, India
}

\begin{abstract}
Several well-known statistical measures similar to LMC and Fisher-Shannon complexity have been computed for confined hydrogen atom in both position $(r)$ and momentum $(p)$ spaces. Further, a more generalized form of these quantities with Rényi entropy $(R)$ is explored here. The role of scaling parameter in the exponential part is also pursued. $R$ is evaluated taking order of entropic moments $\alpha, \beta$ as $\left(\frac{2}{3}, 3\right)$ in $r$ and $p$ spaces. Detailed systematic results of these measures with respect to variation of confinement radius $r_{c}$ is presented for low-lying states such as, $1 s-3 d, 4 f$ and $5 g$. For nodal states, such as $2 s, 3 s$ and $3 p$, as $r_{c}$ progresses there appears a maximum followed by a minimum in $r$ space, having certain values of the scaling parameter. However, the corresponding p-space results lack such distinct patterns. This study reveals many other interesting features.
\end{abstract}

PACS: 03.65-w, 03.65Ca, 03.65Ta, 03.65.Ge, 03.67-a.

Keywords: $L M C$ complexity, FS complexity, Confined hydrogen atom.

\footnotetext{
${ }^{*}$ Corresponding author. Email: akroy@iiserkol.ac.in, akroy6k@gmail.com.
} 


\section{INTRODUCTION}

Quantum particles experience intense changes in their physical and chemical properties under spatial confinement. Spherically confined quantum systems have been explored extensively [1], with wide-spread applications in quantum dot, quantum wells and quantum wires, etc. Dramatic changes in various observable properties such as energy spectrum, transition frequencies, transition probabilities, polarizability, chemical reactivity, ionization potential etc., were reported to occur under such situations [1, 2].

Recently there has been a growing interest in using statistical quantities namely, Fisher information $(I)$, Onicescu energy $(E)$, Shannon entropy $(S)$ and Rényi entropy $(R)$ as descriptors of certain chemical, physical properties of a quantum system. Along these lines, complexity, another relevant concept, is directly related to aforementioned measures, representing their combined effect. A universal characterization has not been possible, but can be proposed as an indicator of pattern, structure or correlation associated with the distribution function in a given system. It depends on the scale of observation, and constitutes an important area of research with contemporary interest in disordered systems, spatial patterns, language, multi-electronic systems, molecular or DNA analysis, social science, [3] 6] etc.

An atom is a complex system; restricting its motion in an enclosure makes it even more fascinating according to a complex world [7, 8]. Complexity, in a system, arises due to breakdown of certain symmetry rules. For finite complexity, the system is either in a state having some less than maximal order or not in equilibrium. Stated differently, it vanishes at two limiting cases, viz., when it is (a) at equilibrium (maximum disorder) or b) completely ordered (maximum distance from equilibrium) [8, 9]. It gives a qualitative idea of organization in a system and is considered as a general indicator of structure and correlation. In literature various definitions are available; some of them are Shiner, Davidson, Landsberg $(S D L)\left\lfloor 10\right.$ 12], López-Ruiz, Mancini, Calbet (LMC) shape $\left(C_{L M C}\right)$ 13-16], Fisher-Shannon $\left(C_{I S}\right)$ [17, 18], Cramér-Rao [18 20] or Generalized Rényi-like complexity [21 23], etc.

The statistical measure of complexity, in product form, can be written as,

$$
C_{L M C}=H . D
$$

where $H$ represents the information content and $D$ gives an idea of concentration of spatial distribution. In order to satisfy conditions such as reaching minimal values for both extremely ordered and disordered limits, invariance under scaling, translation and replication, 
this quantity was criticized [24] and modified [25], giving rise to the expression,

$$
C_{L M C}=D \cdot e^{S}
$$

Principally this gives an interplay between information stored in a system, and measure of a probabilistic hierarchy amongst its observed parts. It has application in diverse fields like detection of periodic, quasi-periodic, linear stochastic, chaotic dynamics [13, 26, 27].

In information theory $E$ signifies a measure of order, because it becomes minimum at equilibrium. Whereas, information entropies like $S, R$, being maximum at equilibrium, signify disorder. Complexity identifies the extent of balance between order and disorder. Sometimes, $I$ is used in place of $E$. So far $S$ has been extensively used as disorder parameter. $C_{I S}$ is another measure, obtained by replacing the pre-exponential global factor in $C_{L M C}$ by a local factor like $I$. It combines global and local characters while preserving desirable properties of complexity. Usefulness of $C_{I S}$ can be judged by looking at the numerous works done for both free and confined atomic systems, like atomic shell structure, ionization process [17, 18, 20, 28] etc. A more generalized version was also proposed that uses $R$ in place of $S$, in $C_{L M C}$ and $C_{I S}$ [29]. Later, a scaling factor (b) was introduced in exponential part.

About a decade ago, $C_{L M C}$ was used in the context of Rydberg states of free hydrogen atom (FHA) in $r, p$ spaces [30]. Later, $C_{L M C}$ and SDL complexity was employed in atoms [8]. However, in a confined hydrogen atom (CHA) complexity measures have been pursued rather rarely. Two major works in this direction involved calculation of $C_{I S}$ in composite space for ground state of CHA under soft and hard confinement [31, 32]. In this endeavor, our focus is to explore four different types of complexity arising out of two order $(I, E)$ and two disorder $(S, R)$ parameters, in both space as functions of confinement radius $\left(r_{c}\right)$. As in the literature [8], we also adopt two $b$ values $\left(\frac{2}{3}\right.$ for $C_{I S}, 1$ for $\left.C_{L M C}\right)$. All calculations were done using the exact wave functions of CHA in $r$ space. The $p$-space wave function is obtained from numerical Fourier transform of $r$-space counterpart. In the end, pilot calculation are done for eight low-lying states viz., $1 s-3 d, 4 f, 5 \mathrm{~g}$. Organization of this article is as follows. Section II gives a brief account of the theoretical method used; Sec. III presents a detailed discussion on our results, while we conclude with a few remarks in Sec. IV. 


\section{METHODOLOGY}

Exact radial wave function for a CHA can be expressed as [33],

$$
\psi_{n, l}(r)=N_{n, l}\left(2 r \sqrt{-2 \mathcal{E}_{n, l}}\right)^{l}{ }_{1} F_{1}\left[\left(l+1-\frac{1}{\sqrt{-2 \mathcal{E}_{n, l}}}\right),(2 l+2), 2 r \sqrt{-2 \mathcal{E}_{n, l}}\right] e^{-r \sqrt{-2 \mathcal{E}_{n, l}}} .
$$

Here, $N_{n, l}$ denotes normalization constant and $\mathcal{E}_{n, l}$ corresponds to energy of a given state characterized by radial and angular quantum numbers $n, l$ respectively, whereas ${ }_{1} F_{1}[a, b, r]$ represents confluent hypergeometric function. Allowed energies are obtained by imposing the boundary condition $\psi_{n, \ell}(0)=\psi_{n, \ell}\left(r_{c}\right)=0$. In this work, generalized pseudospectral (GPS) method was employed to estimate $\mathcal{E}_{n, l}$ of these states. This method has provided very accurate results for various model and real systems including atoms, molecules, some of which could be found in the references [34-36].

The $p$-space wave function is obtained from Fourier transform of $r$-space counterpart,

$$
\begin{aligned}
\psi_{n, l}(p) & =\frac{1}{(2 \pi)^{\frac{3}{2}}} \int_{0}^{\infty} \int_{0}^{\pi} \int_{0}^{2 \pi} \psi_{n, l}(r) \Theta(\theta) \Phi(\phi) e^{i p r \cos \theta} r^{2} \sin \theta \mathrm{d} r \mathrm{~d} \theta \mathrm{d} \phi \\
& =\frac{1}{2 \pi} \sqrt{\frac{2 l+1}{2}} \int_{0}^{\infty} \int_{0}^{\pi} \psi_{n, l}(r) P_{l}^{0}(\cos \theta) e^{i p r \cos \theta} r^{2} \sin \theta \mathrm{d} r \mathrm{~d} \theta .
\end{aligned}
$$

Here $\psi(p)$ is not normalized and needs to be normalized. Integrating over $\theta$ and $\phi$ yields,

$$
\psi_{n, l}(p)=(-i)^{l} \int_{0}^{\infty} \frac{\psi_{n, l}(r)}{p} f(r, p) \mathrm{d} r .
$$

Depending on $l$, this can be rewritten in following simplified form ( $m$ starts with 0$)$,

$$
\begin{aligned}
& f(r, p)=\sum_{k=2 m+1}^{m<\frac{l}{2}} a_{k} \frac{\cos p r}{p^{k} r^{k-1}}+\sum_{j=2 m}^{m=\frac{l}{2}} b_{j} \frac{\sin p r}{p^{j} r^{j-1}}, \quad \text { for even } l, \\
& f(r, p)=\sum_{k=2 m}^{m=\frac{l-1}{2}} a_{k} \frac{\cos p r}{p^{k} r^{k-1}}+\sum_{j=2 m+1}^{m=\frac{l-1}{2}} b_{j} \frac{\sin p r}{p^{j} r^{j-1}}, \quad \text { for odd } l .
\end{aligned}
$$

The values of coefficients $a_{k}, b_{j}$ of even- $l$ and odd- $l$ states can easily be computed from Eq. (2). Normalized position and momentum electron densities are expressed as,

$$
\rho(\mathbf{r})=\left|\psi_{n, l, m}(\mathbf{r})\right|^{2}, \quad \Pi(\mathbf{p})=\left|\psi_{n, l, m}(\mathbf{p})\right|^{2}
$$

Without any loss of generality, let us define complexity in following general form $C=A e^{b . B}$. The order $(A)$ and disorder parameters $(B)$ may include $(E, I)$ and $(R, S)$ 
respectively. With this in mind, we are interested in the following four quantities,

$$
C_{E R}=E e^{b R}, \quad C_{I R}=I e^{b R}, \quad C_{E S}=E e^{b S}, \quad C_{I S}=I e^{b S} .
$$

Shannon entropies of a continuous density distribution are written as ('t' stands for total),

$$
S_{\mathbf{r}}=-\int_{\mathcal{R}^{3}} \rho(\mathbf{r}) \ln [\rho(\mathbf{r})] \mathrm{d} \mathbf{r} ; \quad S_{\mathbf{p}}=-\int_{\mathcal{R}^{3}} \Pi(\mathbf{p}) \ln [\Pi(\mathbf{p})] \mathrm{d} \mathbf{p} ; \quad S_{t}=S_{\mathbf{r}}+S_{\mathbf{p}}
$$

Similarly, Rényi entropies of order $\alpha(\neq 1)$ are obtained by taking logarithm of $\alpha$ and $\beta$-order entropic moments in respective spaces,

$$
R_{\mathbf{r}}^{\alpha}=\frac{1}{1-\alpha} \ln \left(\int_{\mathcal{R}^{3}} \rho^{\alpha}(\mathbf{r}) \mathrm{d} \mathbf{r}\right) ; \quad R_{\mathbf{p}}^{\beta}=\frac{1}{1-\beta} \ln \left[\int_{\mathcal{R}^{3}} \Pi^{\beta}(\mathbf{p}) \mathrm{d} \mathbf{p}\right] ; \quad R_{t}=R_{\mathbf{r}}^{\alpha}+R_{\mathbf{p}}^{\beta} .
$$

The general form of $I_{\mathbf{r}}, I_{\mathbf{p}}$ for a particle in a central potential may be simplified as [37],

$$
I_{\mathbf{r}}=4\left\langle p^{2}\right\rangle-2(2 l+1)|m|\left\langle r^{-2}\right\rangle ; \quad I_{\mathbf{p}}=4\left\langle r^{2}\right\rangle-2(2 l+1)|m|\left\langle p^{-2}\right\rangle ; \quad I_{t}=I_{\mathbf{r}} I_{\mathbf{p}} .
$$

Finally, $E$ is given by the following expressions in conjugate space,

$$
E_{\mathbf{r}}=\int_{\mathcal{R}^{3}} \rho^{2}(\mathbf{r}) \mathrm{d} \mathbf{r} ; \quad E_{\mathbf{p}}=\int_{\mathcal{R}^{3}} \Pi^{2}(\mathbf{p}) \mathrm{d} \mathbf{p} ; \quad E_{t}=E_{\mathbf{r}} E_{\mathbf{p}}
$$

\section{RESULT AND DISCUSSION}

At first let us clear a few things before we begin our discussion. All the tables and figures that follow quote the net information measures in conjugate $r$ and $p$ space of CHA, which may be partitioned in to radial and angular contributions. In a given space, all results correspond to net measures including the angular parts. By squeezing the radial boundary of FHA from infinity to a finite region, one progresses to a CHA. As this does not alter the angular boundary conditions, angular portion of the information measures in FHA and CHA remains unchanged in both spaces. Further as we are solely interested in radial confinement, same will also not change as one modifies $r_{c}$ values from one to another. However, there will be non-vanishing contribution from $l, m$ quantum numbers. Throughout our calculation, magnetic quantum number $m$ is set to 0 . All the aforementioned measures of Eq. (8) have been investigated with respect to $r_{c}$, for two selected values of $b\left(1, \frac{2}{3}\right)$; these are the ones which are widely used in literature. Note that, for $b=1, C_{E S}^{(2)}$ reduces to $C_{L M C}$; similarly $C_{I S}^{(1)}$ corresponding to $b=\frac{2}{3}$ refers to $C_{I S}$ of literature. In order to facilitate the discussion, a 

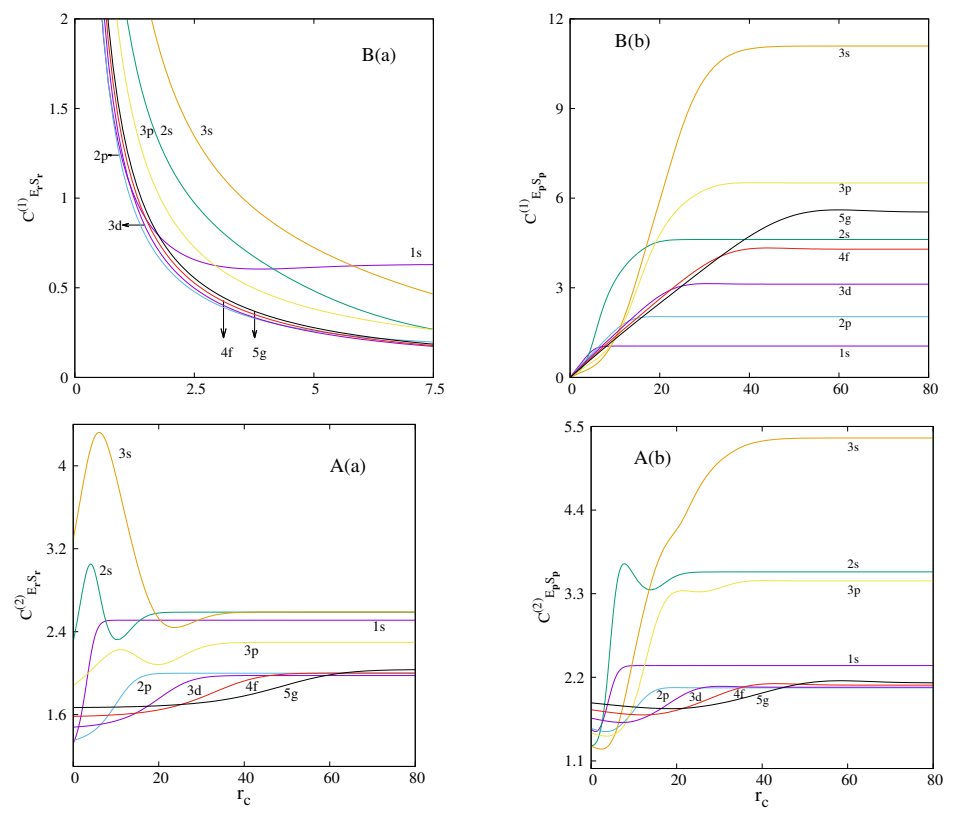

FIG. 1: Variation of $C_{E_{\mathbf{r}} S_{\mathbf{r}}}^{(2)}, C_{E_{\mathbf{p}} S_{\mathbf{p}}}^{(2)}$ (bottom row A) and $C_{E_{\mathbf{r}} S_{\mathbf{r}}}^{(1)}, C_{E_{\mathbf{p}} S_{\mathbf{p}}}^{(1)}$ (top row B) in CHA with $r_{c}$ for $1 s-3 d, 4 f$ and $5 g$ states. See text for details.

few words may be devoted to the notation followed. A uniform symbol $C_{\text {order }_{s}, \text { disorder }_{s}}^{b}$ is used; where the two subscripts refer to two order $(E, I)$ and disorder $(S, R)$ parameters. Another subscript $s$ is used to specify the space; viz., $r, p$ or $t$ (total). Two scaling parameters $b=\frac{2}{3}, 1$ are identified with superscripts 1,2 . These measures are offered systematically for $1 s-3 d$ as well as $4 f$ and $5 g$ states in conjugate spaces, with $r_{c}$ varying in the range of 0.1-100 a.u.

At first, in Fig. $1 C_{E_{\mathbf{r}} S_{\mathbf{r}}}^{(2)}, C_{E_{\mathbf{p}} S_{\mathbf{p}}}^{(2)}$ are plotted against $r_{c}$ in two bottom panels $\mathrm{A}(\mathrm{a})$ and $\mathrm{A}(\mathrm{b})$; two similar plots for $C_{E_{\mathbf{r}} S_{\mathbf{r}}}^{(1)}, C_{E_{\mathbf{p}} S_{\mathbf{p}}}^{(1)}$ in top two segments B(a) and B(b). Note that the range of $r_{c}$ is same for all four panels except $\mathrm{B}(\mathrm{a})$. Panel A(a) clearly reveals that, $C_{E_{\mathbf{r}} S_{\mathbf{r}}}^{(2)}$ for circular states $(1 s, 2 p, 3 d, 4 f, 5 g)$ gradually increases with rise of $r_{c}$ before reaching a threshold corresponding to the FHA result. The particular $r_{c}$ at which this limiting value is reached tends to grow as $l$ goes up. On the other hand, the same for nodal states $(2 s, 3 s, 3 p)$ shows a maximum followed by a minimum with $r_{c}$ and finally converges to respective FHA value. Appearance of such extrema in $C_{E_{\mathbf{r}} S_{\mathbf{r}}}^{(2)}$ thus can be considered as an indication of presence of nodes. Importantly, however, an increase in number of nodes in wave function apparently does not affect the number of extrema produced in $C_{E_{\mathbf{r}} S_{\mathbf{r}}}^{(2)}$. In strong confinement region $\left(r_{c} \lesssim 0.4\right)$, for a particular $r_{c}, C_{E_{\mathbf{r}} S_{\mathbf{r}}}^{(2)}$ enhances with $n$ for the circular states. But in the higher $r_{c}$ region, significant crossing occurs amongst these states; so this ordering is 
TABLE I: $C_{E_{\mathbf{r}} S_{\mathbf{r}}}^{(2)}, C_{E_{\mathbf{p}} S_{\mathbf{p}}}^{(2)}$ and $C_{E_{t} S_{t}}^{(2)}$ for $1 s, 2 s, 2 p, 3 d$ states in CHA at various $r_{c}$.

\begin{tabular}{|c|c|c|c|c|c|c|c|}
\hline$r_{c}$ & $C_{E_{\mathbf{r}} S_{\mathbf{r}}}^{(2)}$ & $C_{E_{\mathbf{p}} S_{\mathbf{p}}}^{(2)}$ & $C_{E_{t} S_{t}}^{(2)}$ & $r_{c}$ & $C_{E_{\mathbf{r}} S_{\mathbf{r}}}^{(2)}$ & $C_{E_{\mathbf{p}} S_{\mathbf{p}}}^{(2)}$ & $C_{E_{t} S_{t}}^{(2)}$ \\
\hline \multicolumn{4}{|c|}{$1 s$} & \multicolumn{4}{|c|}{$2 s$} \\
\hline 0.1 & 1.330123 & 1.5122 & 2.0114 & 0.1 & 2.325609 & 1.2984 & 3.0196 \\
\hline 2.0 & 1.624390 & 1.5304 & 2.4860 & 1.5 & 2.636436 & 1.3766 & 3.6295 \\
\hline 3.5 & 2.012256 & 1.7709 & 3.5636 & 4.1 & 3.051717 & 2.3753 & 7.2489 \\
\hline 4.5 & 2.254326 & 1.9863 & 4.4778 & 6.9 & 2.624591 & 3.6385 & 9.5496 \\
\hline 7.5 & 2.501324 & 2.3284 & 5.8242 & 10.0 & 2.324233 & 3.5457 & 8.2410 \\
\hline 10.0 & 2.510443 & 2.3533 & 5.9078 & 13.0 & 2.401653 & 3.3610 & 8.0721 \\
\hline 16.0 & 2.510692 & 2.3543 & 5.9110 & 38.0 & 2.588344 & 3.5864 & 9.2830 \\
\hline 20.0 & 2.510692 & 2.3543 & 5.9110 & 40.0 & 2.588344 & 3.5864 & 9.2830 \\
\hline \multicolumn{4}{|c|}{$2 p$} & \multicolumn{4}{|c|}{$3 d$} \\
\hline 0.1 & 1.352613 & 1.5276 & 2.0662 & 0.1 & 1.479327 & 1.6617 & 2.4582 \\
\hline 2.5 & 1.386951 & 1.4907 & 2.0676 & 10.0 & 1.539579 & 1.6231 & 2.4989 \\
\hline 5.0 & 1.449780 & 1.5013 & 2.1766 & 16.0 & 1.644781 & 1.7746 & 2.9189 \\
\hline 8.5 & 1.609479 & 1.6609 & 2.6733 & 20.0 & 1.753859 & 1.9185 & 3.3648 \\
\hline 12.0 & 1.821269 & 1.8978 & 3.4565 & 22.0 & 1.811337 & 1.9824 & 3.5909 \\
\hline 14.0 & 1.912716 & 1.9966 & 3.8189 & 26.0 & 1.904810 & 2.0619 & 3.9276 \\
\hline 33.0 & 1.999251 & 2.0613 & 4.1211 & 56.0 & 1.977128 & 2.0698 & 4.0923 \\
\hline 40.0 & 1.999251 & 2.0613 & 4.1211 & 60.0 & 1.977128 & 2.0698 & 4.0923 \\
\hline
\end{tabular}

no longer maintained. On the other hand, at a fixed low $r_{c}, C_{E_{\mathbf{r}} S_{\mathbf{r}}}^{(2)}$ for $2 s, 3 s, 3 p$ states accelerate with number of nodes. When they have equal number of nodes then the state with lower $n$ has greater complexity. But at weak confinement region this ordering dissolves. Now, from panel A(b) one infers that, for all these reported states there occur a minimum in $C_{E_{\mathbf{p}} S_{\mathbf{p}}}^{(2)}$. Position of this minimum shifts toward right with increase in both $n$ and $l$. After the minimum point, $C_{E_{\mathbf{p}} S_{\mathbf{p}}}^{(2)}$ for node-less states grows up to reach their terminal value. Whereas for states having nodes $(2 s, 3 s, 3 p)$, the minimum is preceded by a maximum with increment of $r_{c}$. But importantly, these extrema get flattened with progress of $n$ and fall of $l$ for a given $n$. Now, panel $\mathrm{A}(\mathrm{c})$ in Fig. S1 presents variations of the total quantities $C_{E_{t} S_{t}}^{(2)}$ for the concerned states. As usual the nodeless states $1 s, 2 p, 3 d, 4 f, 5 g$ continually increase with $r_{c}$ until converging to FHA limit, whereas nodal states go through some extrema before reaching that limit-qualitatively much similar to a pattern encountered in $\mathrm{A}(\mathrm{a})$. Next, panels $\mathrm{B}(\mathrm{a})$ and $\mathrm{B}(\mathrm{b})$ in the top row, delineate that, for all these states $C_{E_{\mathbf{r}} S_{\mathbf{r}}}^{(1)}$ monotonically decline and $C_{E_{\mathbf{p}} S_{\mathbf{p}}}^{(1)}$ enhance with rise of $r_{c}$ respectively. However, panel B(c) in Fig. S1 shows that, $C_{E_{t} S_{t}}^{(1)}$ for circular states elevate with $r_{c}$. But, for for the nodal states $(2 s, 3 s, 3 p)$, there occur some extrema, which thinning out with progress in $n, l$ quantum numbers. From the 

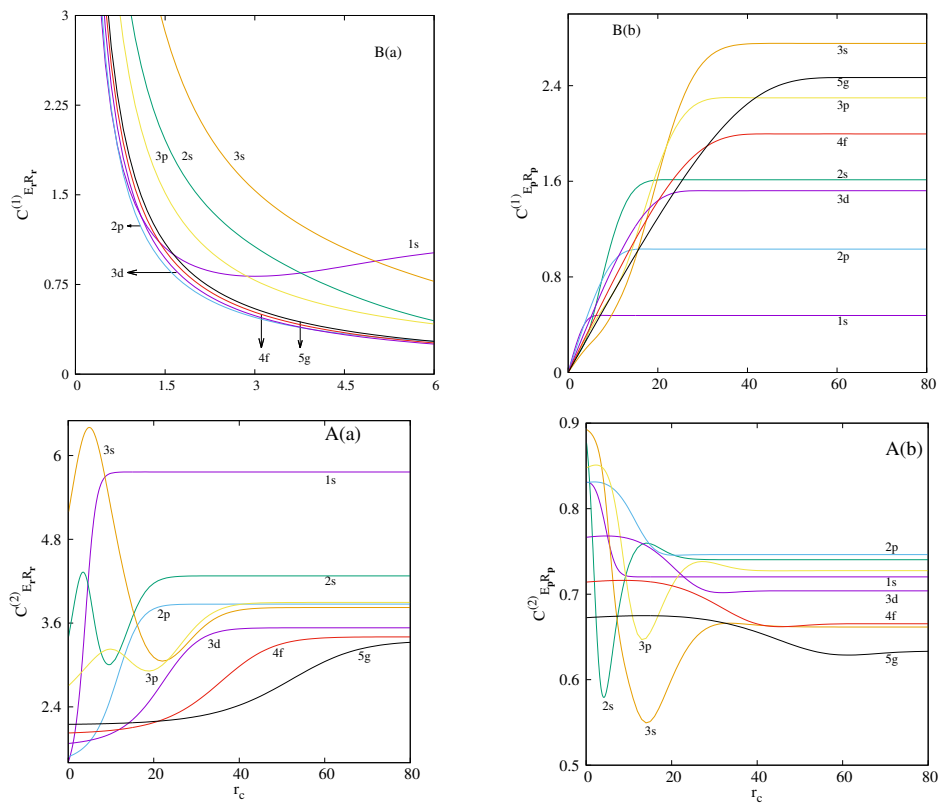

FIG. 2: Changes in $C_{E_{\mathbf{r}} R_{\mathbf{r}}}^{(2)}, C_{E_{\mathbf{p}} R_{\mathbf{p}}}^{(2)}$ (bottom row A) and $C_{E_{\mathbf{r}} R_{\mathbf{r}}}^{(1)}, C_{E_{\mathbf{p}} R_{\mathbf{p}}}^{(1)}$ (top row B) in CHA with $r_{c}$ for $1 s-3 d, 4 f$ and $5 g$ states. For more details, see text.

study of these two sets of complexity measures, namely, $C_{E S}^{(2)}$ in $\mathrm{A}(\mathrm{a})-\mathrm{A}(\mathrm{c})$ and $C_{E S}^{(1)}$ in $\mathrm{B}(\mathrm{a})$ $\mathrm{B}(\mathrm{c})$, it is evident that $C_{E S}^{(2)}$ provides better insight about CHA. Hence, we have presented $C_{E_{\mathbf{r}} S_{\mathbf{r}}}^{(2)}, C_{E_{\mathbf{p}} S_{\mathbf{p}}}^{(2)}, C_{E_{t} S_{t}}^{(2)}$ at some selected $r_{c}$ (not same for all states) in Table I, for $1 s, 2 s, 2 p, 3 d$, while the remaining states $(3 s, 3 p, 4 f, 5 g)$ are offered in Table S1. These results corroborate the conclusions drawn from Figs. 1 and S1. None of these could be directly compared with literature data, as no such works exist, to the best of our knowledge.

Similarly, bottom row of Fig. 2 illustrates the behavior of $C_{E_{\mathbf{r}} R_{\mathbf{r}}}^{(2)}, C_{E_{\mathbf{p}} R_{\mathbf{p}}}^{(2)}$ with changes in $r_{c}$ for the same states of Fig. 1. Panel $\mathrm{A}(\mathrm{a})$ shows that, like $C_{E_{\mathbf{r}} S_{\mathbf{r}}}^{(2)}$, here also $C_{E_{\mathbf{r}} R_{\mathbf{r}}}^{(2)}$ for circular states advances to their respective limiting values with growth of $r_{c}$. The nodal states $(2 s, 3 s, 3 p)$ once again display similar pattern as in $C_{E_{\mathbf{r}} S_{\mathbf{r}}}^{(2)}$. At first they cross through a maximum followed by a minimum before eventually coalescing to the respective FHA values. In stronger confinement region, $\left(r_{c} \lesssim 0.4\right)$, at a certain $r_{c}, C_{E_{\mathbf{r}} R_{\mathbf{r}}}^{(2)}$ enhances with $n$ for the circular states. But for higher $r_{c}$ (FHA limit), there is a decrement in the same for these five states with betterment of $n$. From panel A(b) it is vivid that, for all these circular states, $C_{E_{\mathbf{p}} S_{\mathbf{p}}}^{(2)}$ diminish with $r_{c}$, then attains a shallow minimum and finally stretches to respective FHA value. But for $2 s, 3 s, 3 p$ states, prominent minimum pursued by small maximum are observed (before reaching FHA value). Position of these extrema get right 
TABLE II: $C_{E_{\mathbf{r}} R_{\mathbf{r}}}^{(2)}, C_{E_{\mathbf{p}} R_{\mathbf{p}}}^{(2)}$ and $C_{E_{t} R_{t}}^{(2)}$ for $1 s, 2 s, 2 p, 3 d$ states in CHA at various $r_{c}$.

\begin{tabular}{llll|llll}
\hline \hline$r_{c}$ & $C_{E_{\mathbf{r}} R_{\mathbf{r}}}^{(2)}$ & $C_{E_{\mathbf{p}} R_{\mathbf{p}}}^{(2)}$ & $C_{E_{t} R_{t}}^{(2)}$ & $r_{c}$ & $C_{E_{\mathbf{r}} R_{\mathbf{r}}}^{(2)}$ & $C_{E_{\mathbf{p}} R_{\mathbf{p}}}^{(2)}$ & $C_{E_{t} R_{t}}^{(2)}$ \\
\hline 0.1 & $1 s$ & & & & $2 s$ & & \\
1.5 & 1.62388648 & 0.83076854 & 1.34907381 & 0.1 & 3.40812187 & 0.876978 & 2.988848 \\
3.3 & 3.00911324 & 0.82750748 & 1.66255624 & 2.0 & 4.07095921 & 0.707115 & 2.878639 \\
4.5 & 4.06621535 & 0.80540213 & 2.46953640 & 3.5 & 4.32946637 & 0.589721 & 2.553178 \\
6.0 & 5.08995204 & 0.74682789 & 3.80131819 & 9.6 & 3.00374145 & 0.727635 & 2.185627 \\
18.0 & 5.76509705 & 0.72020586 & 4.15205673 & 30.0 & 4.27524770 & 0.740362 & 3.165232 \\
25.0 & 5.76468568 & 0.72020460 & 4.15175319 & 42.0 & 4.27629214 & 0.740292 & 3.165706 \\
30.0 & 5.76468568 & 0.72020460 & 4.15175319 & 50.0 & 4.27629299 & 0.740292 & 3.165706 \\
\hline & & $2 p$ & & & & $3 d$ & \\
\hline 0.1 & 1.68688780 & 0.830403 & 1.400796 & 0.1 & 1.875596 & 0.766519 & 1.437680 \\
6.2 & 2.00463718 & 0.823814 & 1.651448 & 9.5 & 2.002374 & 0.765598 & 1.533015 \\
9.9 & 2.51770021 & 0.800244 & 2.014775 & 20.0 & 2.565092 & 0.733376 & 1.881177 \\
12.0 & 2.91667206 & 0.781037 & 2.278030 & 25.0 & 3.004262 & 0.711700 & 2.138136 \\
15.0 & 3.43010804 & 0.757407 & 2.597988 & 35.0 & 3.472244 & 0.702409 & 2.438936 \\
40.0 & 3.87153850 & 0.746249 & 2.889133 & 50.0 & 3.531830 & 0.703914 & 2.486106 \\
50.0 & 3.87153994 & 0.746249 & 2.889134 & 70.0 & 3.532549 & 0.703904 & 2.486576 \\
60.0 & 3.87153994 & 0.746249 & 2.889134 & 80.0 & 3.532549 & 0.703904 & 2.486576 \\
\hline \hline
\end{tabular}

shifted with $n$. Moreover, the depth of the minimum enhances with rise of $n$ within a fixed $l$. The relevant total measures are again displayed in Fig. S2 of supplementary material. For nodeless states, $C_{E_{t} R_{t}}^{(2)}$, in panel A(c), like $C_{E_{t} S_{t}}^{(2)}$ advances to their FHA value with progression in $r_{c}$, while for $2 s, 3 s, 3 p$ it passes through a maximum and a minimum before reaching their the same limit. Now panels $\mathrm{B}(\mathrm{a})$ and $\mathrm{B}(\mathrm{b})$ in top row indicate that, for all these states $C_{E_{\mathbf{r}} R_{\mathbf{r}}}^{(1)}$ gradually decline and $C_{E_{\mathbf{p}} R_{\mathbf{p}}}^{(1)}$ show opposite trend with advances in $r_{c}$. However, panel $\mathrm{B}(\mathrm{c})$ of Fig. S2 imprints that, $C_{E_{t} R_{t}}^{(1)}$ for circular states improves with $r_{c}$. But for nodal $(2 s, 2 p, 3 s)$ states it reaches the FHA threshold by passing consecutive maximum and a minimum. This once again suggests that out of $C_{E R}^{(2)}$ and $C_{E R}^{(1)}$, the former offers more detailed knowledge about CHA, which justifies the quantities produced in Table II, namely, $C_{E_{\mathbf{r}} R_{\mathbf{r}}}^{(2)}, C_{E_{\mathbf{p}} R_{\mathbf{p}}}^{(2)}$ and $C_{E_{t} R_{t}}^{(2)}$. These are given for four states $(1 s, 2 s, 2 p, 3 d)$ at eight suitably chosen $r_{c}$ (not same for all states); Table S2 presents same for $3 s, 3 p, 4 f, 5 g$ states. These two Tables II and S2 complement the inferences drawn from Figs. 2 and S2. As in the previous table, here also no literature results could be quoted. Additionally, in $r$ and $p$ spaces $C_{E_{\mathbf{r}} R_{\mathbf{r}}}^{(2)}$ and $C_{E_{\mathbf{p}} R_{\mathbf{p}}}^{(2)}$ exhibit opposite behavior but for $C_{E_{\mathbf{r}} S_{\mathbf{r}}}^{(2)}$ and $C_{E_{\mathbf{p}} S_{\mathbf{p}}}^{(2)}$ an analogous trend is observed. Hence, $C_{E_{\mathbf{r}} R_{\mathbf{r}}}^{(2)}$ 

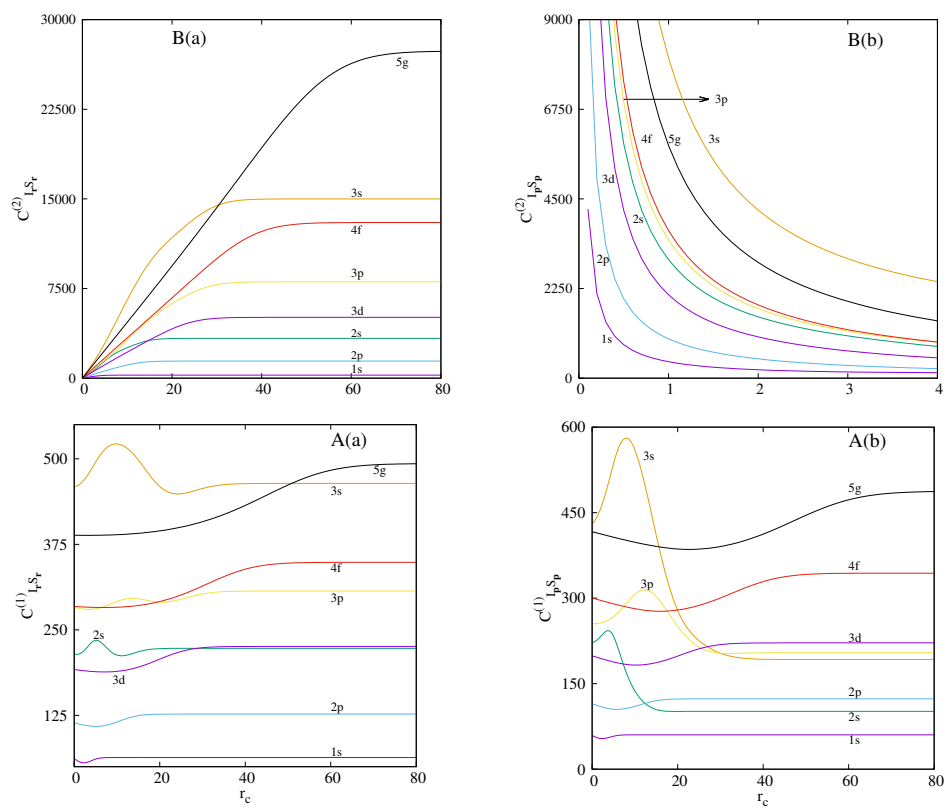

FIG. 3: Variation of $C_{I_{\mathbf{r}} S_{\mathbf{r}}}^{(1)}, C_{I_{\mathbf{p}} S_{\mathbf{p}}}^{(1)}$ (bottom row A) $C_{I_{\mathbf{r}} S_{\mathbf{r}}}^{(2)}, C_{I_{\mathbf{p}} S_{\mathbf{p}}}^{(2)}$ (top row B) in CHA with $r_{c}$ for $1 s-3 d, 4 f$ and $5 g$ states. Consult text for more details.

and $C_{E_{\mathbf{p}} R_{\mathbf{p}}}^{(2)}$ turn out to be a relatively better measure of complexity.

Next in Fig. 3 the two rows incorporating panels $\{\mathrm{A}(\mathrm{a}), \mathrm{A}(\mathrm{b})\},\{\mathrm{B}(\mathrm{a}), \mathrm{B}(\mathrm{b})\}$ portray the variation of $\left\{C_{I_{\mathbf{r}} S_{\mathbf{r}}}^{(1)}, C_{I_{\mathbf{p}} S_{\mathbf{p}}}^{(1)}\right\}$ and $\left\{C_{I_{\mathbf{r}} S_{\mathbf{r}}}^{(2)}, C_{I_{\mathbf{p}} S_{\mathbf{p}}}^{(2)}\right\}$ with changes in $r_{c}$. The bottom two panels as well as A(c) of Fig. S3 illustrate that, for circular states, $C_{I_{\mathbf{r}} S_{\mathbf{r}}}^{(1)}, C_{I_{\mathbf{p}} S_{\mathbf{p}}}^{(1)}$ and $C_{I_{t} S_{t}}^{(1)}$ initially fall with growth of $r_{c}$, then attain a minimum and finally converge to respective FHA values. But, for the other three states $(2 s, 3 s, 3 p), C_{I_{\mathbf{r}} S_{\mathbf{r}}}^{(1)}$ grows to a maximum then falls down to a minimum with advance in $r_{c}$ and eventually join the FHA result. Whereas, $C_{I_{\mathbf{p}} S_{\mathbf{p}}}^{(1)}$ and $C_{I_{t} S_{t}}^{(1)}$, for these three non-circular states only pass through a maximum before reaching their borderline values. On the other hand, top panels $\mathrm{B}(\mathrm{a})$ and $\mathrm{B}(\mathrm{b})$ portray that, for all eight states considered, $C_{I_{\mathbf{r}} S_{\mathbf{r}}}^{(2)}$ and $C_{I_{\mathbf{p}} S_{\mathbf{p}}}^{(2)}$ consistently progress and reduce respectively with increase in $r_{c}$. However, panel $\mathrm{B}(\mathrm{c})$ in Fig. S3 shows that, for circular states $C_{I_{t} S_{t}}^{(2)}$ enhances with development of $r_{c}$ and for states with nodes, it passes through a maximum before merging to FHA result. A careful study of Figs. 3 and S3 reveals another interesting feature that, in case of CHA, $C_{I_{\mathbf{r}} S_{\mathbf{r}}}^{(1)}, C_{I_{\mathbf{p}} S_{\mathbf{p}}}^{(1)}, C_{I_{t} S_{t}}^{(1)}$ provides a more detailed account than $C_{I_{\mathbf{r}} S_{\mathbf{r}}}^{(2)}, C_{I_{\mathbf{p}} S_{\mathbf{p}}}^{(2)}, C_{I_{t} S_{t}}^{(2)}$. Thus, to get a quantitative idea, $C_{I_{\mathbf{r}} S_{\mathbf{r}}}^{(1)}, C_{I_{\mathbf{p}} S_{\mathbf{p}}}^{(1)}, C_{I_{t} S_{t}}^{(1)}$ values at some selected $r_{c}$ 's are given in Tables III $(1 s, 2 s, 2 p, 3 d)$ and $\mathrm{S} 3(3 s, 3 p, 4 f, 5 g)$. Again no results are available in literature except the lone ground state for $C_{I S}^{(1)}$ at few $r_{c}$ values, which are 
TABLE III: $C_{I_{\mathbf{r}} S_{\mathbf{r}}}^{(1)}, C_{I_{\mathbf{p}} S_{\mathbf{p}}}^{(1)}$ and $C_{I_{t} S_{t}}^{(1)}$ for $1 s, 2 s, 2 p, 3 d$ states in CHA at selected $r_{c}$.

\begin{tabular}{llcl|llll}
\hline \hline$r_{c}$ & $C_{I_{\mathbf{r}} S_{\mathbf{r}}}^{(1)}$ & $C_{I_{\mathbf{p}} S_{\mathbf{p}}}^{(1)}$ & $C_{I_{t} S_{t}}^{(1)}$ & $r_{c}$ & $C_{I_{\mathbf{r}} S_{\mathbf{r}}}^{(1)}$ & $C_{I_{\mathbf{P}} S_{\mathbf{p}}}^{(1)}$ & $C_{I_{t} S_{t}}^{(1)}$ \\
\hline $0.1^{a}$ & 61.445391 & 58.4510 & 3591.5482 & 0.1 & 214.635698 & 221.5739 & 47557.6874 \\
$1.0^{b}$ & 57.747939 & 55.7023 & 3216.6965 & 0.7 & 214.216370 & 224.3204 & 48053.1193 \\
2.3 & 55.670758 & 53.7138 & 2990.2906 & 3.0 & 224.290385 & 240.5022 & 53942.3512 \\
4.0 & 58.707169 & 55.8278 & 3277.4955 & 5.2 & 234.766329 & 229.7707 & 53942.4448 \\
4.7 & 60.463080 & 57.2340 & 3460.5492 & 11.0 & 212.022314 & 126.2148 & 26760.3588 \\
6.5 & 62.907313 & 59.6342 & 3751.4320 & 17.0 & 220.839663 & 101.9061 & 22504.9152 \\
$30.0^{c}$ & 63.398969 & 60.3065 & 3823.3703 & 43.0 & 223.025525 & 101.3888 & 22612.3074 \\
$40.0^{d}$ & 63.398969 & 60.3065 & 3823.3703 & 50.0 & 223.025525 & 101.3889 & 22612.3209 \\
\hline & & & & & $3 d$ & \\
\hline 0.1 & 114.078664 & 114.3281 & 13042.3998 & 0.1 & 191.947614 & 198.3921 & 38080.8988 \\
2.5 & 110.537817 & 108.7010 & 12015.5737 & 3.0 & 189.827438 & 192.0738 & 36460.8929 \\
5.1 & 108.960438 & 105.0430 & 11445.5361 & 7.2 & 188.511787 & 184.6662 & 34811.7628 \\
7.1 & 110.071219 & 105.5415 & 11617.0842 & 12.0 & 191.035748 & 183.2506 & 35007.4345 \\
9.5 & 114.166616 & 109.1567 & 12462.0605 & 17.0 & 199.745109 & 190.8078 & 38112.9401 \\
15.0 & 124.642286 & 120.1211 & 14972.1809 & 21.0 & 209.748916 & 201.1179 & 42184.2742 \\
34.0 & 126.882910 & 123.4402 & 15662.4635 & 56.0 & 225.764533 & 221.5783 & 50024.5236 \\
50.0 & 126.882916 & 123.4403 & 15662.4772 & 70.0 & 225.764533 & 221.5783 & 50024.5236 \\
\hline \hline
\end{tabular}

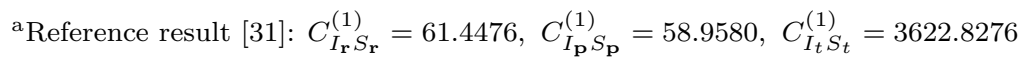

${ }^{\mathrm{b}}$ Reference result 31]: $C_{I_{\mathrm{r}} S_{\mathrm{r}}}^{(1)}=57.7561, C_{I_{\mathrm{p}} S_{\mathrm{p}}}^{(1)}=55.6956, C_{I_{t} S_{t}}^{(1)}=3216.7606$

${ }^{\mathrm{c}}$ Reference result 31): $C_{I_{\mathrm{r}} S_{\mathbf{r}}}^{(1)}=63.4008, C_{I_{\mathrm{p}} S_{\mathrm{p}}}^{(1)}=60.3087, C_{I_{t} S_{t}}^{(1)}=3823.6198$

${ }^{\mathrm{d}}$ Reference result [31]: $C_{I_{\mathbf{r}} S_{\mathbf{r}}}^{(1)}=63.4008, C_{I_{\mathbf{p}} S_{\mathbf{p}}}^{(1)}=60.3087, C_{I_{t} S_{t}}^{(1)}=3823.6198$

${ }^{\dagger}$ Reference values are multiplied with a $8 \pi^{2} e$ factor in both $r$ and $p$ space.

duly quoted for comparison (for $r_{c}=0.1,1,30,40$ ). Current results are in good agreement with the reported one and may be useful for future references.

Finally, in Fig. 4 the two lower $(\mathrm{A}(\mathrm{a}), \mathrm{A}(\mathrm{b}))$ and upper $(\mathrm{B}(\mathrm{a})-\mathrm{B}(\mathrm{b}))$ panels depict the alteration of our last complexity measure, viz., $C_{I_{\mathbf{r}} R_{\mathbf{r}}}^{(1)}, C_{I_{\mathbf{p}} R_{\mathbf{p}}}^{(1)}$ and $C_{I_{\mathbf{r}} R_{\mathbf{r}}}^{(2)}, C_{I_{\mathbf{p}} R_{\mathbf{p}}}^{(2)}$ with variation in $r_{c}$. Here, again the bottom row as well as panel $\mathrm{A}(\mathrm{c})$ of Fig. S4 shows that, for circular states, $C_{I_{\mathbf{r}} R_{\mathbf{r}}}^{(1)}, C_{I_{\mathbf{p}} R_{\mathbf{p}}}^{(1)}$ and $C_{I_{t} R_{t}}^{(1)}$ reduce to attain a minimum with rise of $r_{c}$ and finally assume the fate of FHA. But for three remaining nodal states, $C_{I_{\mathbf{r}} R_{\mathbf{r}}}^{(1)}$ progress via a maximum and minimum successively with growth in $r_{c}$; after that they approach the FHA result. However, $C_{I_{\mathbf{p}} R_{\mathbf{p}}}^{(1)}$ and $C_{I_{t} R_{t}}^{(1)}$ for the aforesaid states with nodes, climb a maximum and fall down asymptotically to a constant FHA value. Besides these, panels B(a) and B(b) portray that, for all these reported eight states concerned, $C_{I_{\mathbf{r}} R_{\mathbf{r}}}^{(2)}$ and $C_{I_{\mathbf{p}} R_{\mathbf{p}}}^{(2)}$ rise and fall respectively 

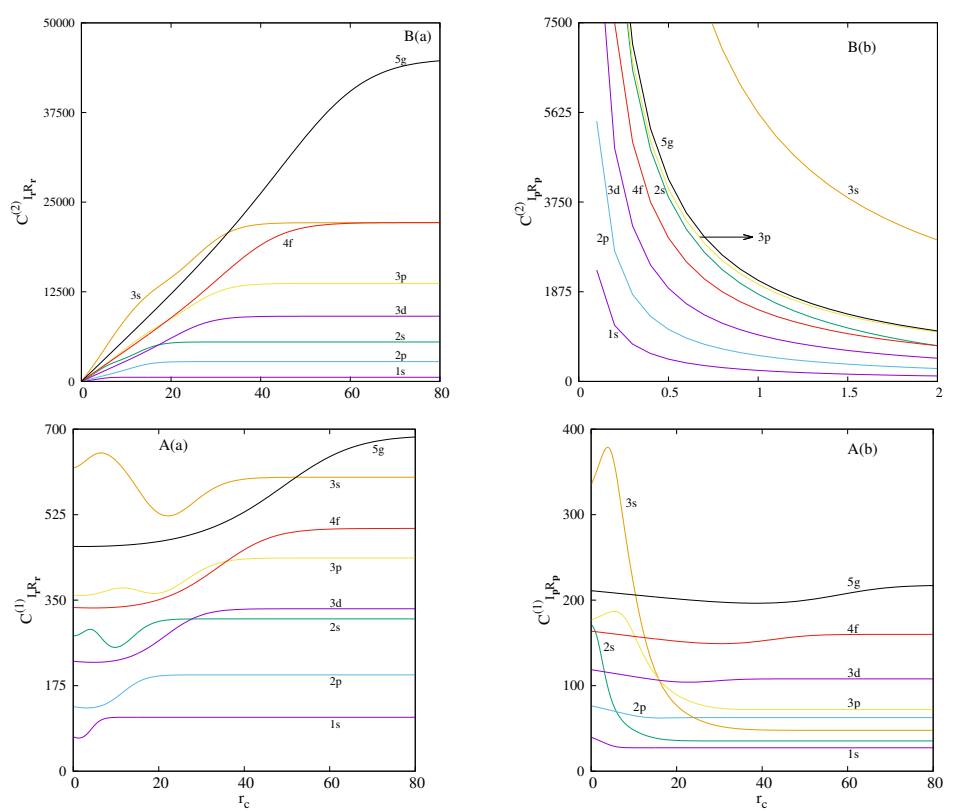

FIG. 4: Plots of $C_{I_{\mathbf{r}} R_{\mathbf{r}}}^{(1)}, C_{I_{\mathbf{p}} R_{\mathbf{p}}}^{(1)}$ (bottom row A) and $C_{I_{\mathbf{r}} R_{\mathbf{r}}}^{(2)}, C_{I_{\mathbf{p}} R_{\mathbf{p}}}^{(2)}$ (top row B) in CHA with $r_{c}$ for $1 s-3 d, 4 f$ and $5 g$ states. For further details, see text.

with growth of $r_{c}$. However, panel $\mathrm{B}(\mathrm{c})$ in Fig. $\mathrm{S} 4$ shows that, for circular states $C_{I_{t} R_{t}}^{(2)}$ improve with elevation of $r_{c}$ and for $2 s, 3 s, 3 p$ states it proceeds through a maximum before reaching the limiting value at FHA. A closer investigation of Figs. 4 and S4 conveys that, $C_{I_{\mathbf{r}} R_{\mathbf{r}}}^{(1)}, C_{I_{\mathbf{p}} R_{\mathbf{p}}}^{(1)}, C_{I_{t} R_{t}}^{(1)}$ scale the system of this orientation better than $C_{I_{\mathbf{r}} R_{\mathbf{r}}}^{(2)}, C_{I_{\mathbf{p}} R_{\mathbf{p}}}^{(2)}, C_{I_{t} R_{t}}^{(2)}$. Hence, to conclude, the former three measures are offered in Tables IV and S4, at some appropriately chosen $r_{c}$. No comparison could be made due to lack of any literature data and hopefully these would be useful in future.

\section{FUTURE AND OUTLOOK}

Various complexity measures like $C_{E S}, C_{I S}, C_{E R}, C_{I R}$ are explored for low-lying states of CHA in both $r$ and $p$ space, keeping $m$ fixed at zero. We have pursued our calculation using both global quantity $(E)$ and local quantity $(I)$ as a measure of order in a system. Except for some results of $C_{I S}$ in ground state, all these quantities are reported here for the first time. It is found that, $C_{E S}^{(2)}, C_{E R}^{(2)}$ offer more detailed explanation than $C_{E S}^{(1)}, C_{E R}^{(1)}$ about the system. On the contrary, $C_{I S}^{(1)}, C_{I R}^{(1)}$ interpret the behavior of CHA more efficiently than that of $C_{I S}^{(2)}, C_{I R}^{(2)}$. Hence, depending upon the nature of complexity measures, it is necessary to determine the appropriate value of $b$. Accurate results for $C_{E S}^{(2)}, C_{E R}^{(2)}, C_{I S}^{(1)}, C_{I R}^{(2)}$ 
TABLE IV: $C_{I_{\mathbf{r}} R_{\mathbf{r}}}^{(1)}, C_{I_{\mathbf{p}} R_{\mathbf{p}}}^{(1)}$ and $C_{I_{t} R_{t}}^{(1)}$ for $1 s, 2 s, 2 p, 3 d$ states in CHA at some selected $r_{c}$.

\begin{tabular}{llll|llll}
\hline \hline$r_{c}$ & $C_{I_{\mathbf{r}} R_{\mathbf{r}}}^{(1)}$ & $C_{I_{\mathbf{p}} R_{\mathbf{p}}}^{(1)}$ & $C_{I_{t} R_{t}}^{(1)}$ & $r_{c}$ & $C_{I_{\mathbf{r}} R_{\mathbf{r}}}^{(1)}$ & $C_{I_{\mathbf{p}} R_{\mathbf{p}}}^{(1)}$ & $C_{I_{t} R_{t}}^{(1)}$ \\
\hline 0.1 & $1 s$ & & & & $2 s$ & \\
1.0 & 70.18835424 & 39.206889 & 2751.867082 & 0.1 & 276.919929 & 170.569679 & 47234.143661 \\
2.5 & 70.98469720 & 34.502985 & 2449.183973 & 4.0 & 290.232344 & 96.448638 & 27992.514409 \\
5.0 & 93.90104729 & 29.677676 & 2786.764914 & 7.0 & 268.480761 & 61.038709 & 16387.719163 \\
5.8 & 100.48737687 & 28.715280 & 2885.523167 & 9.8 & 253.236641 & 48.608701 & 12309.504305 \\
6.8 & 105.75702471 & 27.975405 & 2958.595632 & 18.0 & 300.561968 & 36.584635 & 10995.950138 \\
23.0 & 110.34101779 & 27.379301 & 3021.060018 & 50.0 & 311.686645 & 35.411689 & 11037.350749 \\
30.0 & 110.34101779 & 27.379301 & 3021.060018 & 70.0 & 311.686648 & 35.411668 & 11037.344253 \\
\hline & & $2 p$ & & & $3 d$ & \\
\hline 0.1 & 132.174030 & 76.150268 & 10065.087867 & 0.1 & 224.853345 & 118.438787 & 26631.357655 \\
3.4 & 129.499950 & 72.573286 & 9398.237011 & 16.0 & 244.855787 & 106.300730 & 26028.349158 \\
5.8 & 132.007059 & 69.694271 & 9200.135775 & 20.0 & 266.977357 & 104.494337 & 27897.622145 \\
8.2 & 140.266419 & 66.892350 & 9382.750441 & 23.0 & 286.056400 & 104.096971 & 29777.604868 \\
10.0 & 150.439765 & 65.033889 & 9783.683044 & 26.0 & 303.369560 & 104.554144 & 31718.544807 \\
13.0 & 170.727938 & 62.902501 & 10739.214335 & 56.0 & 332.420701 & 107.955748 & 35886.725520 \\
60.0 & 197.127576 & 62.701899 & 12360.273469 & 86.0 & 332.423192 & 107.955697 & 35886.977628 \\
70.0 & 197.127576 & 62.701899 & 12360.273469 & 90.0 & 332.423192 & 107.955697 & 35886.977628 \\
\hline \hline
\end{tabular}

(radial plus angular) are provided for $1 s-3 d, 4 f$ and $5 g$ states of CHA, most of them for the first time. Further, an investigation of all these quantities in the realm of Rydberg states under different kinds of confined environment, as well as the correlation between complexity and periodicity in many-electron atomic systems may be worthwhile pursuing.

\section{ACKNOWLEDGEMENT}

Financial support from DST SERB, New Delhi, India (sanction order: EMR/2014/000838) is gratefully acknowledged. SM is obliged to IISER-K for supporting her JRF. NM thanks DST SERB, New Delhi, India, for a National-post-doctoral fellowship (sanction order: PDF/2016/000014/CS).

[1] J. R. Sabin, E. Brändas and S. A. Cruz (Eds.), The Theory of Confined Quantum Systems, Parts I and II, Advances in Quantum Chemistry, Vols. 57 and 58 (Academic Press, 2009). 
[2] K. D. Sen (Ed.), Electronic Structure of Quantum Confined Atoms and Molecules, (Springer, Switzerland, 2014).

[3] O. A. Rosso, M. T. Martin and A. Plastino, Physica A 320, 497 (2003).

[4] C. R. Shalizi, K. L. Shalizi and R. Haslinger, Phys. Rev. Lett. 93, 118701 (2004).

[5] K. Ch. Chatzisavvas, Ch. C. Moustakidis and C. P. Panos, J. Chem. Phys. 123, 174111 (2005).

[6] P. A. Bouvrie, J. C. Angulo and J. S. Dehesa, Physica A 390, 2215 (2011).

[7] N. Goldenfeld and L. P. Kadanoff, Science 284, 87 (1999).

[8] K. D. Sen (Ed.), Statistical Complexity: Applications in Electronic Structure, (Springer, 2012).

[9] K. D. Sen (Ed.), Reviews of Modern Quantum Chemistry: A Celebration of the Contributions of Robert G. Parr, Vol. 1 (World Scientific, Singapore, 2002).

[10] P. T. Landsberg, Phys. Lett. A 102, 171 (1984).

[11] P. T. Landsberg and J. S. Shiner, Phys. Lett. A 245, 228 (1998).

[12] J. S. Shiner, M. Davison and P. T. Landsberg, Phys. Rev. E 59, 1459 (1999).

[13] R. López-Ruiz, H. L. Mancini and X. Calbet, Phys. Lett. A 209, 321 (1995).

[14] C. Anteneodo and A. R. Plastino, Phys. Lett. A 223, 348 (1996).

[15] R. G. Catalán, J. Garay and R. López-Ruiz, Phys. Rev. E 66, 011102 (2002).

[16] J. R. Sánchez and R. López-Ruiz, Physica A 355, 633 (2005).

[17] K. D. Sen, J. Antolín and J. C. Angulo, Phys. Rev. A 76, 032502 (2007).

[18] J. C. Angulo, J. Antolín and K. D. Sen, Phys. Lett. A 372, 670 (2008).

[19] J. S. Dehesa, P. Sánchez-Moreno and R. J. Yáñez, J. Comput. Appl. Math. 186, 523 (2006).

[20] J. Antolín and J. C. Angulo, Int. J. Quant. Chem. 109, 586 (2009).

[21] X. Calbet, R. López-Ruiz, Phys. Rev. E 63, 066116 (2001).

[22] M. T. Martin, A. Plastino and A. O. Rosso, Physica A 369, 439 (2006).

[23] E. Romera and A. Nagy, Phys. Lett. A 372, 6823 (2009).

[24] D. P. Feldman and J. P. Crutchfield, Phys. Lett. A 238, 244 (1998).

[25] R. López-Ruiz, Biophys. Chem. 115, 215 (2005).

[26] T. Yamano, J. Math. Phys. 45, 1974 (2004).

[27] T. Yamano, Physica A 340, 131 (2004).

[28] J. C. Angulo and J. Antolín, J. Chem. Phys. 128, 164109 (2008).

[29] J. C. Angulo, J. Antolín and R. O. Esquivel, in Statistical Complexity: Applications in Electronic Structure, K. D. Sen (Ed.), pp. 167 (Springer, 2012). 
[30] J. Sañudo and R. López-Ruiz, Phys. Lett. A 372, 5283 (2008).

[31] N. Aquino, A. Flores-Riveros and J. F. Rivas-Silva, Phys. Lett. A 377, 2062 (2013).

[32] Á. Nagy, K. D. Sen and H. E. Montgomery Jr., Phys. Lett. A 373, 2552 (2009).

[33] B. L. Burrows and M. Cohen, Int. J. Quant. Chem. 106, 478 (2006).

[34] A. K. Roy, J. Phys. G 30, 269 (2004).

[35] K. D. Sen and A. K. Roy, Phys. Lett. A 357, 112 (2006).

[36] A. K. Roy, Int. J. Quant. Chem. 115, 937 (2015); ibid., 116, 953 (2016).

[37] E. Romera, P. Sánchez-Moreno and J. S. Dehesa, Chem. Phys. Lett. 414, 468, (2005). 


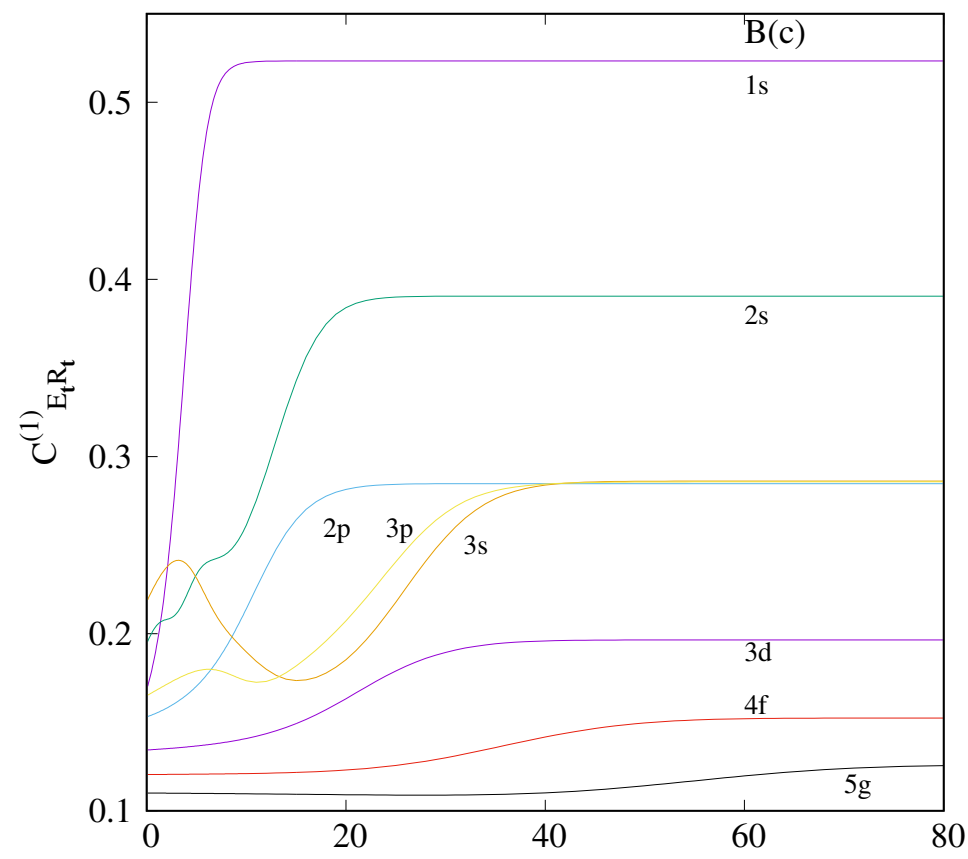




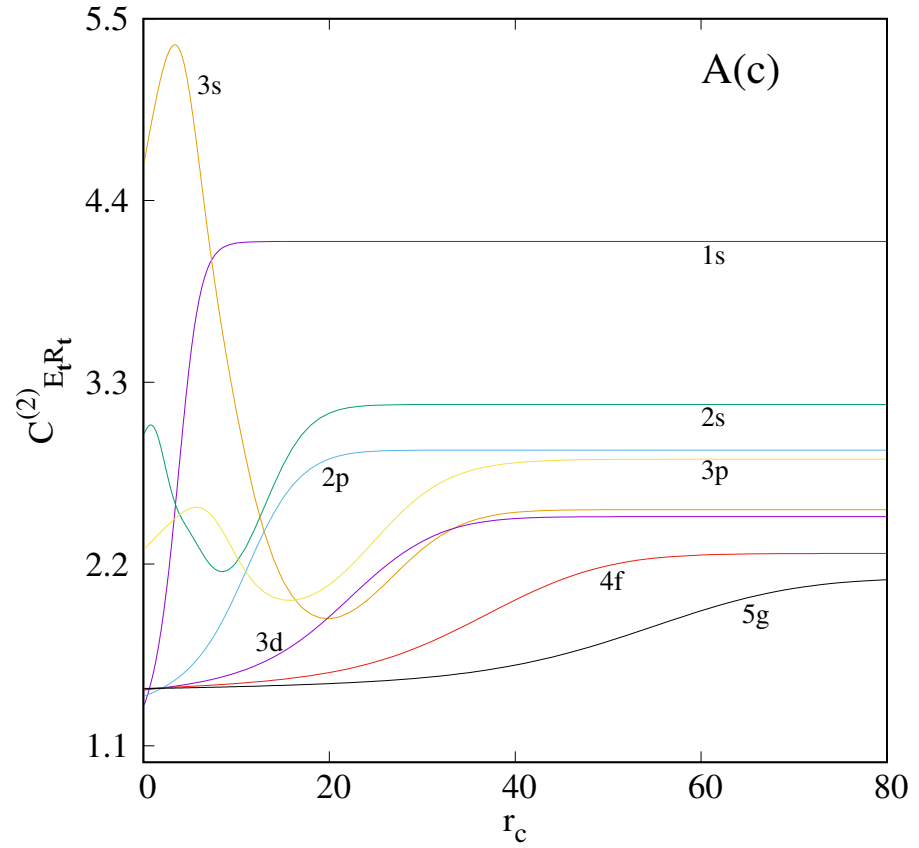




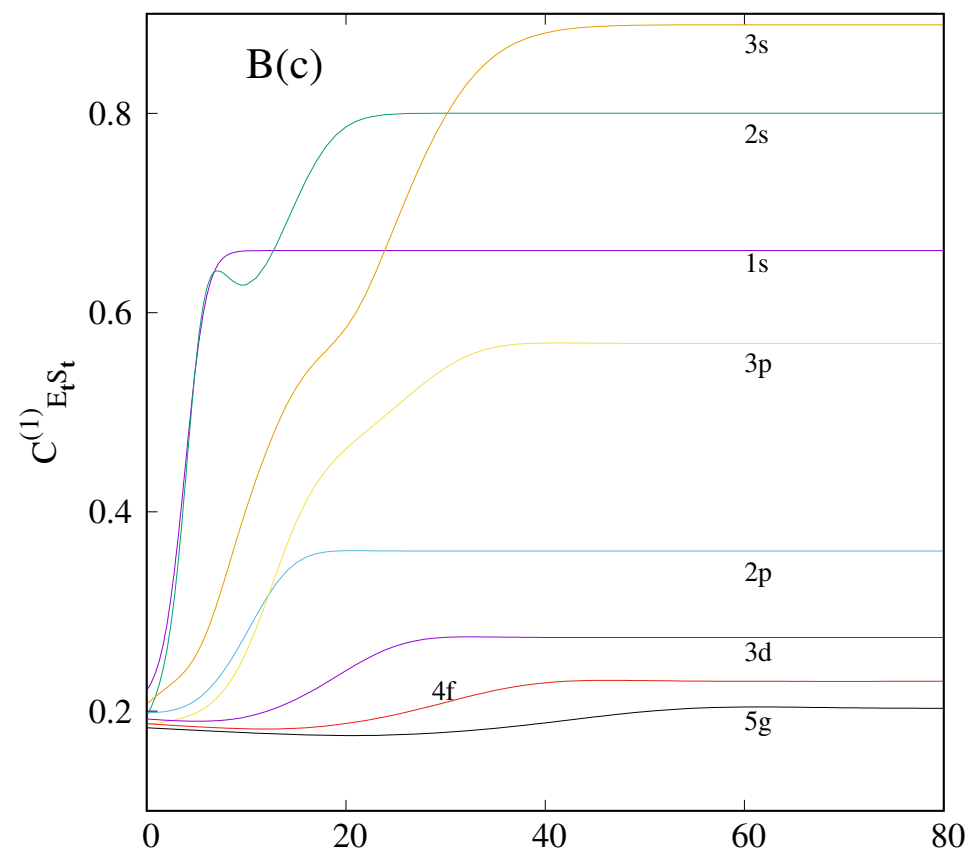




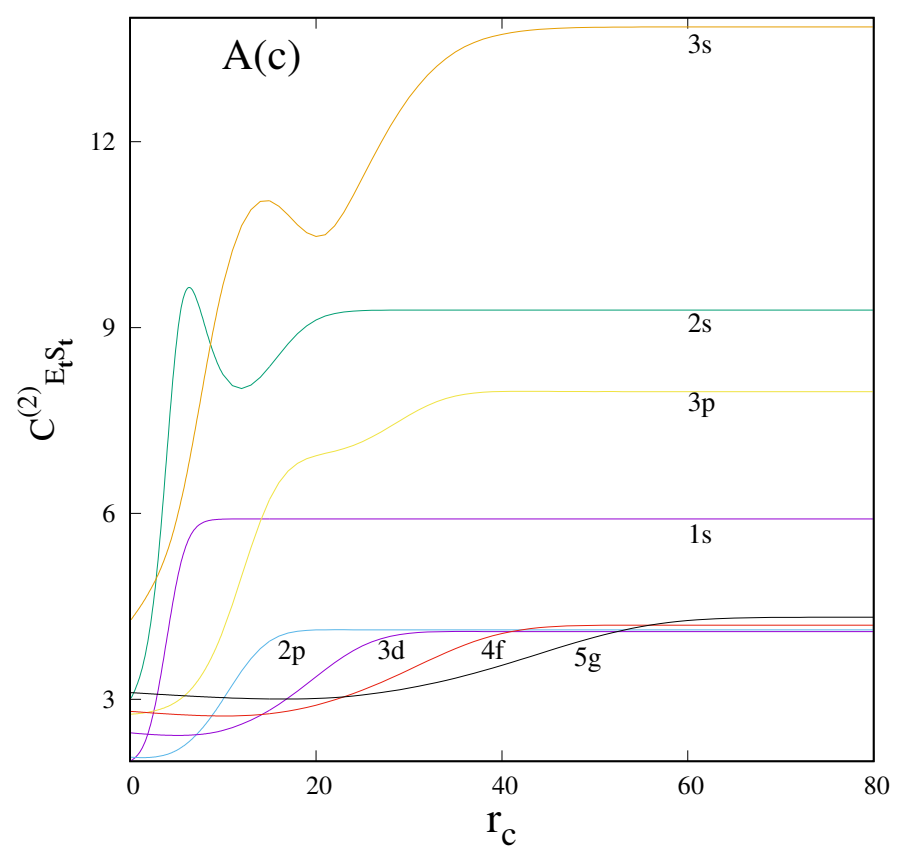




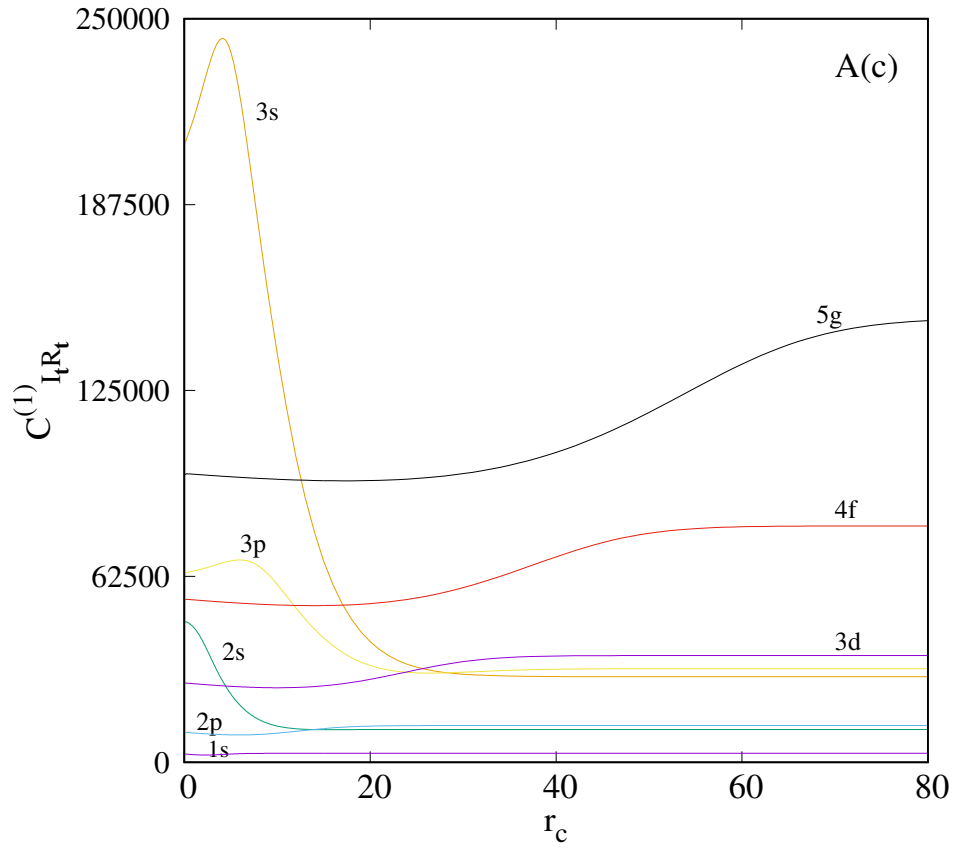




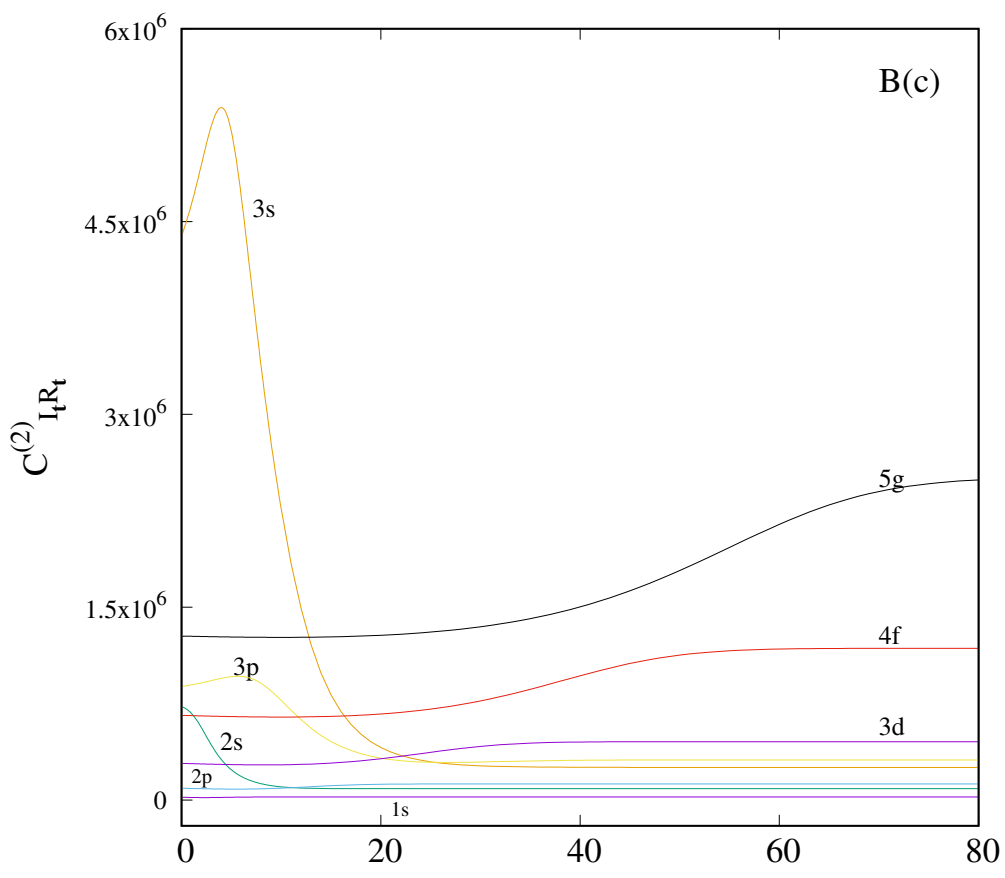




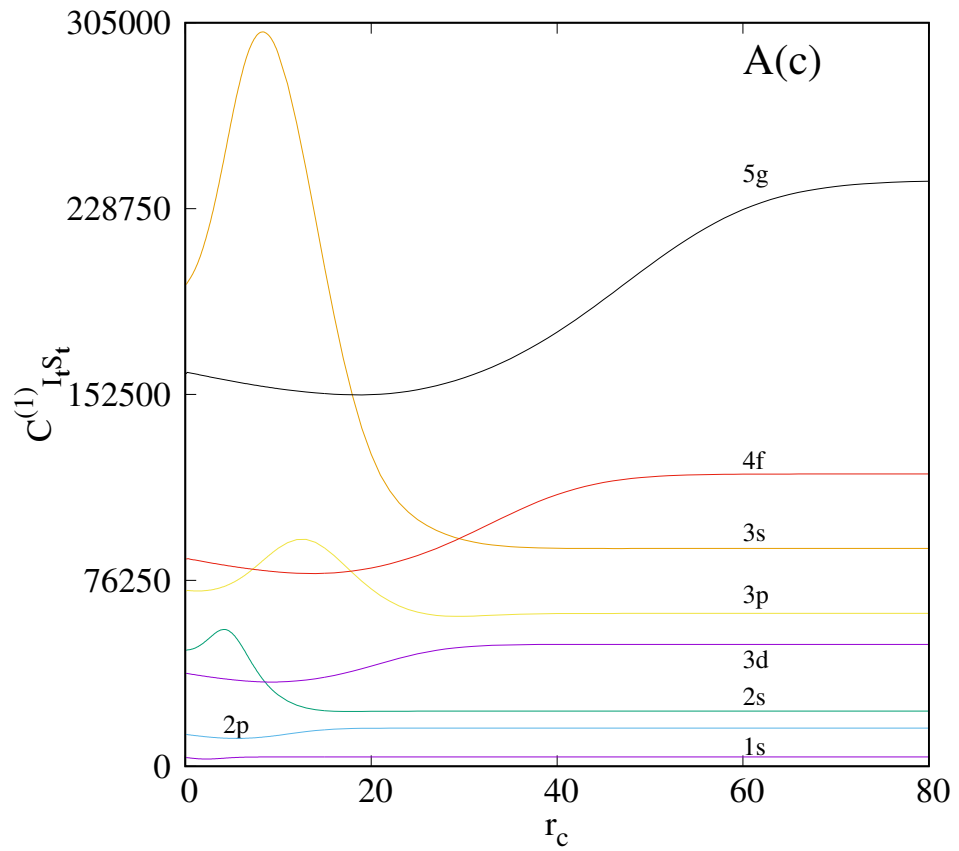




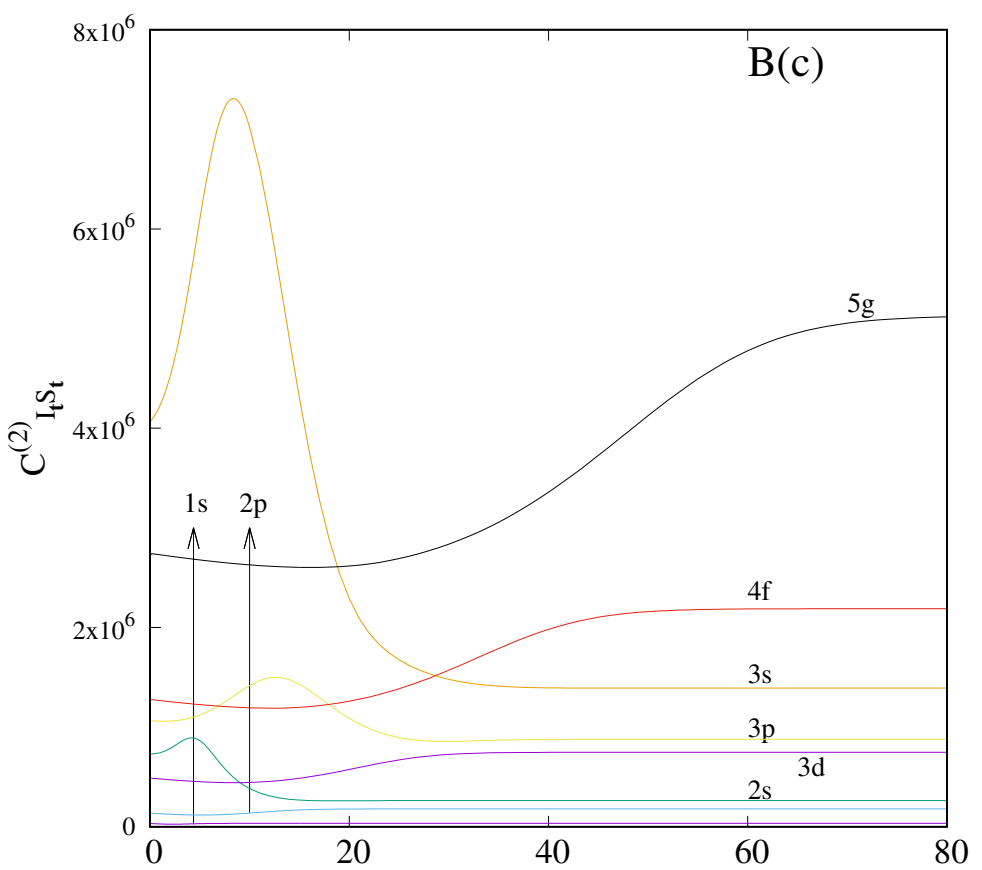

